
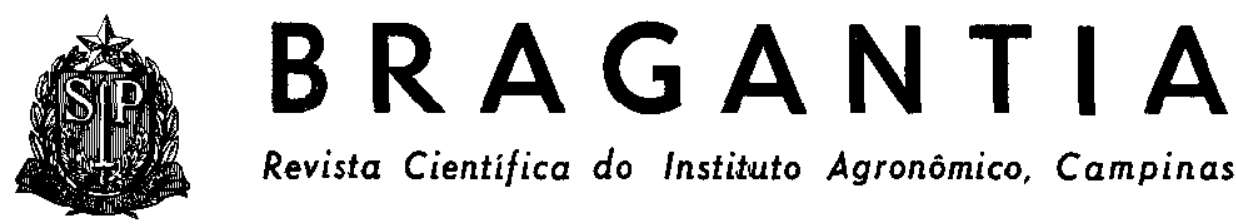

Revista Cientifica do Instituto Agronômico, Campinas

\title{
DELINEAMENTOS "COMPOSTOS CENTRAIS ORTOGONAIS, ROTACIONAIS E DIVISIVEIS EM BLOCOS" (1)
}

\author{
Armando Conagin, Divisão de Plantas Alimenticias Básicas, Instituto Agronômico
}

\section{RESUMO}

Na família dos delineamentos compostos centrais, os compostos centrais ortogonais săo mais eficientes que os outros e a estimação dos coeficientes do modelo é facilmente obtida. Dentro da famillia dos delineamentos compostos centrais ortogonais, os compostos centrais ortogonais rotacionais apresentam algumas vantagens adicionais, principalmente uma uniformidade de variancias para os pontos que se encontram à mesma distância do centro do delineamento. A obtençāo das propriedades ortogonalidade, rotacionalidade e partição em blocos torna o delineamento mais eficiente e aplicável naqueles casos em que possam existir gradientes como o de fertilidade, no caso da experimentação de campo. A partição em dois, três ou cinco blocos é, entăo, altamente desejável. Delineamentos com essas três características foram conseguidos para determinadas combinaçōes de valores, sendo os resultados mais interessantes apresentados no quadro 1 para $k=2,3,4,5,6$ e 7; os resultados que apresentam um asterisco representam delineamentos ortogonais, quase rotacionais e subdivisiveis em blocos, de forma quase perfeitamente ortogonal (as covariancias entre os coeficientes quadráticos puros e os coeficientes de blocos são quase nulas); para fins práticos, esses delineamentos poderiam ser tratados como se fossem ortogonalmente subdivididos em blocos. As soluções obtidas implicam a exigéncia de ser alocado certo número de pontos centrais; esses pontos, por sua vez, possibilitam uma estimativa precisa do erro e o teste de adequaçäo do modelo.

\section{INTRODUÇÃO}

Os delineamentos compostos centrais têm sido bastante utilizados em pesquisas conduzidas em tecnologia industrial, biologia, agronomia, medicina, química etc.
Delineamentos compostos centrais ortogonais têm-se constituído em um competidor dos fatoriais e dos fatoriais fracionados, em pesquisas recentes. Esses delineamentos são bastante flexíveis, possibilitando ao pesquisador, para um número deter-

(1) Trabalho apresentado na 10.a Conferência Internacional de Biometria, Guarujá (SP), em agosto de 1979. Recebido para publicação a 25 de junho de 1980. 
minado de fatores, escolher entre várias alternativas, a que mais lhe convém: dependendo do valor de $\alpha$ escolhido é possível obter-se ortogonalidade, rotacionalidade ou ambas. A ortogonalidade propicia a estimação independente para os coeficientes do modelo e a rotacionalidade proporciona variâncias idênticas para pontos situados à mesma distância do centro, em qualquer direção. Dependendo do número de pontos centrais e feita uma distribuição conveniente. é possível dividir o delineamento em dois, três ou mais blocos, ortogonalmente, sem perder as características atrás mencionadas.

Box, citado por NALIMOV et alii (8), mostrou que é possível construir delineamentos que sejam, ao mesmo tempo, ortogonais e rotacionais. Contudo, segundo o autor citado, é impossível manter as duas propriedades, simultaneamente, para delineamentos de segunda e terceira ordem.

O presente trabalho procurou. não obstante, determinar soluções que possibilitassem a obtenção de delineamentos que fossem, simultaneamente, ortogonais, rotacionais $\mathrm{e}$ subdivisíveis em blocos. Em alguns casos, obtiveram-se soluções exatas; em outros, as soluções foram aproximadas, pois conseguiram-se delineamentos compostos centrais ortogonais quase rotacionais e divisiveis em blocos, de forma praticamente ortogonal.

Esses delineamentos são aplicáveis sobretudo para aqueles casos em que só uma parte das observações pode ser experimentada de cada vez (um bloco por vez, como acontece em certos experimentos industriais) ou nos casos, como na agricultura, em que todo o experimento tem que ser executado ao mesmo tempo, mas se sabe que existem diferenças de fertilidade, havendo vantagem na alocação dos tratamentos em blocos; ainda, em outras áreas, como na experimentação animal, em que os blocos poderiam ser lotes diferentes, raças etc.

\section{MATERIAL E MÉTODO}

\subsection{Generalidades}

O delineamento composto central consta de uma parte fatorial, uma parte axial e pontos centrais (1).

No caso de três fatores, por exemplo, o composto central completo com o menor número possível de pontos, consta de uma parte fatorial $\left(2^{3}=8\right.$ tratamentos nos níveis -1 e +1 , para cada fator), seis pontos na parte axial (nos níveis ${ }^{-} \alpha$ e $+\alpha$ para um dos eixos e no nível zero para os outros dois fatores) e, ainda, um ponto na parte central do delineamento (tratamento 000), abrangendo $2^{3}+2(3)+1=15$ pontos.

Quando o número de fatores $\mathrm{k}$ cresce $(k=4,5,6,7$ etc.), para não crescer demasiadamente 0 número total $\mathrm{N}$ de pontos, usa-se apenas uma parte (fração) da parte fatorial, ou seja $2^{\mathrm{k}-\mathrm{t}}$ pontos (se se utilizar a fração $1 / 4$ do fatorial completo, então, $\mathrm{f}=2$ ), além da parte axial e dos pontos centrais.

Esse tipo de delineamento foi desenvolvido por BOX \& HUNTER (1), BOX \& WILSON (2) e outros, visando inicialmente a sua utilização em pesquisas da indústria, na química 'etc., onde, normalmente, os 
erros experimentais são muito pequenos, o processo é normalmente repetível no espaço e no tempo, podendo as determinações ser feitas sequiencialmente.

A técnica de partição desses delineamentos, em blocos (dois, três ou mais), possibilitou seu uso na Agricultura e em outras áreas da ciência.

$\mathrm{O}$ modelo de segundo grau, para $\mathrm{k}$ fatores, levando em consideração a existência de interações, é o que se segue:

$$
\begin{gathered}
\mathrm{Y}_{\mathrm{u}}=\beta_{0}^{\prime}+{ }_{\mathrm{i}} \stackrel{\mathrm{k}}{=}{ }_{1} \beta_{\mathrm{i}} \mathrm{x}_{\mathrm{iu}}+\mathrm{i}_{\mathrm{i}}^{\stackrel{\mathrm{k}}{=}} \beta_{\mathrm{ii}}\left(\mathrm{x}_{\mathrm{iu}}^{2}-\mathrm{c}\right)+\underset{\mathrm{i}<\mathrm{j}}{\mathrm{\Sigma}} \beta_{\mathrm{ij}} \mathrm{x}_{\mathrm{iu}} \mathrm{x}_{\mathrm{ju}}+\epsilon_{\mathrm{u}} \\
\mathrm{u}=1,2, \ldots, \mathrm{N}
\end{gathered}
$$

No caso, a matriz $\mathrm{X}$ das observações (esquematizadas) para $\mathrm{k}=2$ é a que se segue:

$$
\mathrm{X}=\left[\begin{array}{rrrrrr}
\beta_{0}^{\prime} & \beta_{1} & \beta_{2} & \beta_{11} & \beta_{22} & \beta_{12} \\
1 & -1 & -1 & 1-\mathrm{c} & 1-\mathrm{c} & 1 \\
1 & +1 & -1 & 1-\mathrm{c} & 1-\mathrm{c} & -1 \\
1 & -1 & +1 & 1-\mathrm{c} & 1-\mathrm{c} & -1 \\
1 & +1 & +1 & 1-\mathrm{c} & 1-\mathrm{c} & 1 \\
1 & 0 & 0 & -\mathrm{c} & -\mathrm{c} & 0 \\
1 & -\alpha & 0 & \alpha^{2}-\mathrm{c} & -\mathrm{c} & 0 \\
1 & +\alpha & 0 & \alpha^{2}-\mathrm{c} & -\mathrm{c} & 0 \\
1 & 0 & -\alpha & -\mathrm{c} & \alpha^{2}-\mathrm{c} & 0 \\
\mathrm{~N} & 0 & +\alpha & -\mathrm{c} & \alpha^{2}-\mathrm{c} & 0
\end{array}\right]
$$

A extensão para $\mathrm{k}=3,4, \ldots$ etc. é imediata.

$A$ matriz $\mathrm{A}=\mathrm{X}^{\prime} \mathrm{X}$ para $\mathrm{k}=2$, torna-se então:

$$
X^{\prime} X=\left[\begin{array}{cccccc}
N & & & & & \\
& d & & & & \\
& & d & & & \\
& & p & q & \\
& & q & p & \\
& & & & h
\end{array}\right]
$$

onde: $\mathrm{N}=\mathrm{F}^{\prime}+\mathrm{T} ; \mathrm{d}=\mathrm{F}^{\prime}+2 \alpha^{2}$

$$
\begin{aligned}
& \mathrm{p}=\left[\mathrm{F}^{\prime} \mathrm{T}-4 \mathrm{~F}^{\prime} \alpha^{2}-4 \alpha^{4}+2\left(\mathrm{~F}^{\prime}+\mathrm{T}\right) \alpha^{4}\right] \div\left(\mathrm{F}^{\prime}+\mathrm{T}\right) \\
& \mathrm{q}=\left[\mathrm{F}^{\prime} \mathrm{T}-4 \mathrm{~F}^{\prime} \alpha^{2}-4 \alpha^{4}\right] \div\left(\mathrm{F}^{\prime}+\mathrm{T}\right) \\
& \mathrm{F}^{\prime}=\left[1 / 2^{\mathrm{f}}\right]\left[2^{\mathrm{k}}\right] ; \mathrm{T}=2 \mathrm{k}+\mathrm{n} ; \mathrm{n}=\mathrm{a}_{0}+\mathrm{b}_{0} \\
& \mathrm{~h}=\mathrm{F}^{\prime} ; \mathrm{c}=\left[\mathrm{F}^{\prime}+2 \alpha^{2}\right] \div \mathrm{N} ; \mathrm{p}=\mathrm{q}+2 \alpha^{4}
\end{aligned}
$$


A extensão para $\mathrm{k}=3,4, \ldots$ é imediata.

No caso, $\mathrm{N}$ é o número total de pontos do delineamento, $\mathrm{F}$ ' é o número de pontos da parte fatorial, $2 \mathrm{k}$ o número de pontos da parte axial, $\mathrm{n}$ o número de pontos centrais, sendo $a_{0}$ os pontos centrais da parte axial, $b_{0}$ os pontos centrais da parte fatorial e $\mathrm{f}$ o grau de fracionamento do fatorial.

BOX \& HUNTER (1) e BOX \& WILSON (2) desenvolveram as condições para a obtenção seja dos compostos centrais, seja dos compostos centrais ortogonais, dos compostos centrais rotacionais, e, ainda, as condições necessárias para se conseguir a partição ortogonal, em blocos, do delineamento.

Fazendo-se $\mathrm{q}=0$, as covariâncias entre os coeficientes quadráticos puros ficam eliminadas e o delineamento torna-se ortogonal (a matriz $X^{\prime} X$ passa a ser diagonal). A resolução de $q=0$ (7) leva-nos a obter a equação $\alpha^{4}+F^{\prime} \alpha^{2}-\left(\mathrm{N}-\mathrm{F}^{\prime}\right) \mathrm{F}^{\prime} / 4=0$.

A condição de rotacionalidade implica em que se obtenha $F^{\prime}+2 \alpha^{2}=3 F^{\prime}$ ou $\alpha=\left(F^{\prime}\right)^{1 / 4}(2,7)$.

Para se obter a partição ortogonal em blocos é necessário que cada bloco se constitua em um delineamento de primeira ordem, ortogonal, e, ainda, que a contribuição de cada bloco, para o total da soma de quadrados do delineamento, seja proporcional ao número de unidades existentes dentro de cada bloco $(\mathbf{2}, \mathbf{7})$.

Deve-se ter, por isso, que $2 \alpha^{2} \div\left(\mathrm{F}^{\prime}+2 \alpha^{2}\right)=\left(2 \mathrm{k}+\mathrm{a}_{0}\right) \div \mathrm{N}=\mathrm{n}_{0} \div \mathrm{N}$. Nessa fórmula, $\mathrm{a}_{0}$ representa o número de pontos centrais na parte axial, devendo os restantes $b_{0}=n-a_{0}$ pontos centrais ficar igualmente distribuídos nos outros blocos da parte fatorial.

O modelo completo deve incluir, por isso, os termos correspondentes a blocos, o mesmo acontecendo à matriz $\mathrm{X}$. $\mathrm{O}$ modelo (A) passa a ser:

$$
\begin{aligned}
& \mathrm{Y}_{\mathrm{u}}=\beta_{0}^{\prime}+{ }_{\mathrm{i}}^{\stackrel{\mathrm{k}}{=}}{ }_{1} \beta_{\mathrm{i}} \mathrm{x}_{\mathrm{iu}}+{ }_{\mathrm{i}}^{\stackrel{\mathrm{k}}{=}}, \quad \beta_{\mathrm{ii}}\left(\mathrm{x}_{\mathrm{in}}^{2-} \mathrm{c}\right)+\underset{\mathrm{i}<\mathrm{j}}{\Sigma} \beta_{\mathrm{ij}} \mathrm{x}_{\mathrm{iu}} \mathrm{x}_{\mathrm{ju}}+ \\
& +{ }_{\mathrm{m}}^{\stackrel{\mathrm{b}}{=}} 1^{\delta_{\mathrm{m}}}\left(\mathrm{z}_{\mathrm{nu}}-\overline{\mathrm{z}}_{\mathrm{m}}\right)+\epsilon_{\mathrm{u}} \\
& \text { onde: } \quad \mathrm{u}=1,2, \ldots, \mathrm{N} \\
& \mathrm{i}, \mathrm{j}=1,2, \ldots, \mathrm{k} \\
& \mathrm{m}=1,2, \ldots, \quad \mathrm{b}
\end{aligned}
$$

A maneira de bem utilizar esses delineamentos, alocar os tratamentos, escolher os níveis, proceder aos cálculos e à análise dos resultados experimentais, para os vários tipos de delineamentos compostos centrais, é encontrada em alguns livros de texto, tais como COCHRAN \& COX (3), DAVIES (4) e MYERS (7). 
BOX \& HUNTER (1) e BOX \& WILSON (2) procuraram desenvolver delineamentos do tipo composto central que fossem ortogonais e rotacionais, que fossem ortogonais e subdivisíveis em blocos e rotacionais subdivisíveis em blocos, conciliando as condições de ortogonalidade com rotacionalidade, ortogonalidade com partição em blocos e rotacionalidade com partição em blocos.

MYERS (7) apresenta, para $k$ variando de dois a oito, quadro com valores de $\alpha$ que tornam o composto central ortogonal, quadro com os valores de $\alpha$ que tornam o composto central rotacional, quadro com os delineamentos que são ao mesmo tempo compostos centrais ortogonais e rotacionais e ainda quadro com os delineamentos rotacionais ou quase rotacionais e ortogonalmente subdivisíveis em blocos.

\subsection{Obtenção de delineamentos compostos centrais ortogonais e, simultaneamente, rotacionais ou quase perfeitamente rota- cionais, divisíveis em blocos ou divisíveis, de forma quase com- pletamente ortogonal, em blocos}

Para conseguir delineamentos desse último tipo, o autor procurou impor as três condiçóes apontadas, através do processo descrito a seguir.

Inicia-se pela busca do valor de $\alpha$ que leva à rotacionalidade. Determinado $\alpha$, busca-se o valor que leva à partição em blocos, calculando-se $\left(2 \alpha^{2}\right) \div\left(F^{\prime}+2 \alpha^{2}\right)=n_{0} \div \mathrm{N}=\Omega$. $O$ valor $\mathrm{n}_{0}=\mathrm{N} \Omega$ é substituído na equação

$$
\begin{aligned}
& \mathrm{n}_{0}^{2} \mathrm{q}=4\left(\mathrm{~N}-2 \mathrm{n}_{0}\right) \alpha^{4}-4 \mathrm{~F}^{3} \mathrm{n}_{0} \alpha^{2}+ \\
& +\mathrm{F}^{\prime} \mathrm{n}_{0}^{2}=0
\end{aligned}
$$

que concilia as condições de ortogonalidade com a partição ortogonal em blocos. Determina-se o valor de $\mathrm{N}$ que torna $\mathrm{q}=0$. Se $\mathrm{N}$ for exato, segue-se o processo inverso, chegando-se a $\mathrm{n}_{0}, \alpha$ e à solução desejada.

Há casos, porém, em que o $\mathrm{N}$ obtido é um número não inteiro e $\mathrm{n}_{0}=\mathbf{Q} \mathrm{N}$ também não o é. Nesse caso, tomam-se como solução números inteiros, próximos ao $\mathrm{N}$ e ao $\mathrm{n}_{0}$ obtidos, substituindo-os em (C); daí, recalcula-se o valor $\alpha$ que constitui a estimativa procurada; escolhe-se o par de valores $n_{0}$ e $N$ que proporciona a melhor solução visando à quase perfeita partição em blocos e à quase completa rotacionalidade.

Os valores obtidos para $\mathbf{k}=$ $2,3,4,5,6$ e 7 representando soluções ótimas onde $F$ ' é um fatorial completo ou parte do mesmo, encontram-se no quadro 1 : os valores apresentados com um asterisco são soluções quase ótimas em relação à rotacionalidade e partição quase perfeitamente ortogonal, em blocos.

Segundo LUCAS (6), na comparação de vários delineamentos, pelo critério do $|\mathrm{X} X|$ máximo, a $\mathrm{D}$-eficiência dos delineamentos compostos centrais decresce à medida que cresce o número de fatores $k$. Para $n \geq 3$, o aumento de pontos centrais diminui um pouco a eficiência média geral. Não obstante, segundo aquele autor, os pontos centrais continuam a ser usados, na prática, e muitas vezes são repetidos mais pesadamente que outros pontos do delineamento, pelas seguintes razões: 


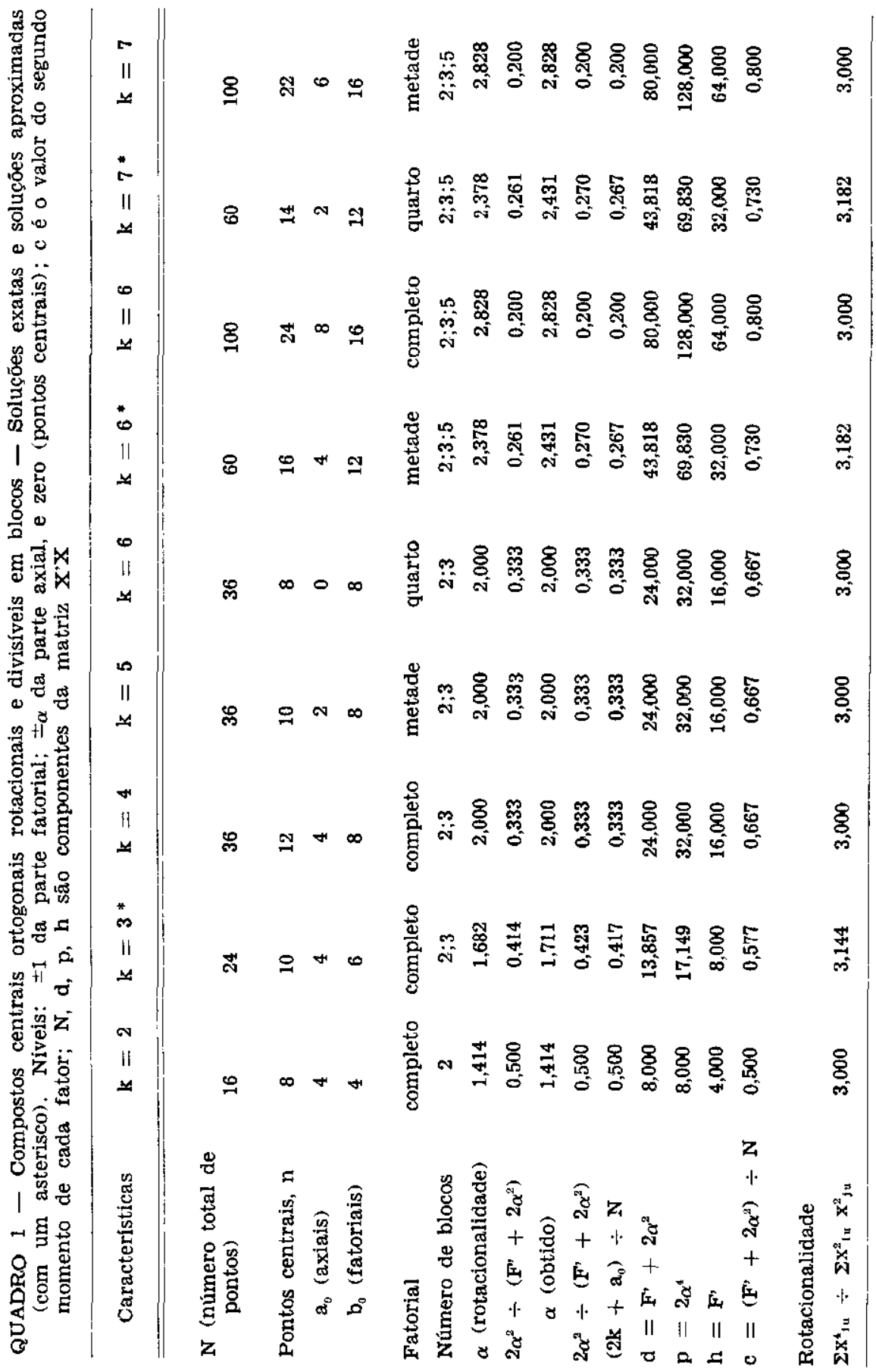


a) Embora $\left|X^{\prime} X\right|$ seja um critério geral bom, outras consideraçöes, como a de se obter uma estimativa do erro, por exemplo, são muito importantes.

b) O pesquisador sabe, a priori, que o ponto central do delineamento está próximo da área mais importante das decisões (ponto extremo, ponto de maior rentabilidade econômica no caso de ensaios de fertilizantes etc.).

c) A adição de pontos centrais a um delineamento composto central aumenta a informação obtida na região, apesar de que pode diminuir a informação por ponto experimental.

No caso dos delineamentos com fracionamento da parte fatorial, em metade ou quarta parte, deve-se escolher a interação ou interações que proporcionam o melhor confundimento, como recomendam DAVIES (4) e HARTLEY (5).

\section{CONCLUSÕES}

Esses delineamentos poderão ser utilizados com sucesso em experimentos como os de adubação, no estudo de $\mathrm{N}, \mathrm{P}$ e $\mathrm{K}$, por exemplo $(\mathrm{k}=3)$, em que se deseja dispor de cinco níveis para cada fator, utilizando um número moderado $\mathrm{N}$ de pontos; convém acentuar que a ortogonalidade, a rotacionalidade e a partição em blocos são propriedades altamente desejáveis (7); ainda, por apresentarem vários pontos centrais, o teste de adequação do modelo pode ser feito, e com precisão razoável.

No caso de experimentos de adubação, deve-se calibrar bem a amplitude das doses escolhidas de forma a que as decisões econômicas ótimas possam estar situadas no intervalo zero a um das doses, mais próximas de zero do que de um.

\section{"ORTHOGCNAL, ROTATABLE AND BLOCKED CENTRAL COMPOSITE" DESIGNS}

\section{SUMMARY}

In the family of central composite designs, the orthogonal central composite is more efficient than the others, and the estimation of the coefficients is easily obtained.

Inside the family of orthogonal central composite designs the rotatable orthogonal central composite have some additional advantages, regarding uniformity of variances of the points at the same distance from the center of the design.

Putting together the conditions for rotatability, orthogonality and blocking, through the choice of an adequate number of points, designs are obtained that satisfy the three specifications and that may be divided in 2,3 or 5 blocks. Some of them represent designs with orthogonality, near rotatability and very near orthogonal blocking (the covariances between the pure quadratic and the block coefficients are so small that, for practical purposes, the design can be analysed as if it was orthogonally blocked)

In fertilizer experiments, orthogonality, rotatability and blocking are very important; the solutions presented may be useful for research workers interested in central composite designs with the characteristics pointed out and that are blockable. They present also the possibility of obtaining a precise estimate of the error and the test of adequacy of the model. 
Vol. 41, Art. n.o 5

\section{REFERENCIAS BIBLIOGRAFICAS}

1. BOX, G. E. P. \& HUNTER, J. S. Multifactor experimental designs for exploring response surfaces. Annals of Mathematical Statistics, 28:195-241, 1957.

2. \& WILSON, K. B. On the experimental attainment of optimum conditions. Journal of the Royal Statistical Society B, 13:1-45, 1951.

3. COCHRAN, W. G. \& COX, G. M. Experimental designs. 2. ed. New York, John Wiley, 1964. 611p.

4. DAVIES, O. L. Design and analysis of industrial experiments. New York, Hafner Publishing Company, 1954. 637p.

5. HARTLEY, H. O. Smallest composite designs for quadratic response surfaces. Biometrics, 15:611-624, 1959.

6. LUCAS, J. M. Optimum composite designs. Technometrics, 16:561-567, 1974.

7. MYERS, R. H. Response surface methodology. Boston, Allyn and Bacon, 1971. 243p.

8. NALIMOV, V. V.; GOLIKOVA, T. I.; MIRESHINA, N. G. On practical use of the concept of D-optimality. Technometrics, 12:799-812, 1970. 